through massive sequencing techniques or using Single-Gene strategies such as Sanger sequencing.

The lack of a cost-effective Gold-Standard has prompted the development of new techniques so that this determination can be carried out routinely in most centers.

In this study we intend to explore the diagnostic accuracy of a novel technology called MODAPLEX that combines multiplex-qPCR and capillary-electrophoresis in order to study POLE mutations in endometrial carcinomas.

Methodology A total of 76 patients diagnosed with endometrial cancer with available histological and molecular classification, were selected, obtaining a paraffin block with adequate viability and tumor representation. From each case, eight sections of $10 \mu \mathrm{m}$ thickness were obtained, subsequently isolating DNA. Those samples with a concentration over $10 \mathrm{ng} / \mu \mathrm{l}$ were tested by MODAPLEX. Any positive result was reconfirmed by Sanger sequencing.

Result(s)* A total of 76 samples were finally submitted to the test: 10 were POLE mutated, $20 \mathrm{CNL}, 29 \mathrm{CNH}$ and $20 \mathrm{MSI}$.

MODAPLEX identified a total of 11 samples with mutations in POLE: V411L(4), P286R(3), S297F(1), A456P(1), $\mathrm{T} 278 \mathrm{M}(1)$ and $\mathrm{L} 424 \mathrm{~V}(1)$. All these mutations were located in the exonuclease domain and had a functional impact on the protein. Ten mutations were confirmed afterwards by Sanger sequencing, except one sample harboring the T278M mutation, which were considered a false positive result of MODAPLEX.

MODAPLEX demonstrated a sensitivity of $100 \%$, a specificity of $98,5 \%$, a Positive Predictive Value of $90,9 \%$ and a Negative of $100 \%$.

Conclusion* MODAPLEX is a promising technology still in development that allows the determination of the main 'Hotspot' mutations in POLE gene in a fast, practical and efficient way.

Following a Single-Gene approach and in this clinical context, this technology could compete with Sanger sequencing for the study of POLE mutations.

This test could emerge as a valid and fast alternative to Next - Generation Sequencing, especially in those centers where they do not have access to massive sequencing techniques. CANCER

${ }^{1} \mathrm{~L}$ Roškar*, ${ }^{2} \mathrm{~T}$ Klančič, ${ }^{2} \mathrm{~S}$ Smrkolj, ${ }^{2} \mathrm{~T}$ Lanisnik Rizner. ${ }^{1}$ Faculty of Medicine, University of Ljubljana, Ljubljana, Slovenia, Department of Obstetrics and Gynecology, , Ljubljana, Slovenia; ${ }^{2}$ Faculty of Medicine, University of Ljubljana, Ljubljana, Slovenia, Institute of Biochemistry, Ljubljana, Slovenia

\subsection{6/ijgc-2021-ESGO.176}

Introduction/Background* Endometrial cancer (EC) is the most common gynecological malignancy in more developed countries. At present, endometrial histology is needed for diagnosis, as there are no valid, accurate noninvasive diagnostic methods. Screening of patients' plasma biomarkers might enable a more precise diagnosis of EC and a tailored treatment approach. The aim of our prospective case-control pilot study was to quantify 37 different angiogenic factors to evalute their potential diagnostic and/or prognostic value.

Methodology 76 postmenopausal women (38 patients with endometrioid EC and 38 control patients with prolapsed uterus or chronic pelvic pain) that underwent surgical treatment at the University Medical Centre Ljubljana were included in this prospective case-control pilot study. Plasma samples were evaluated using commercially available kit that enables

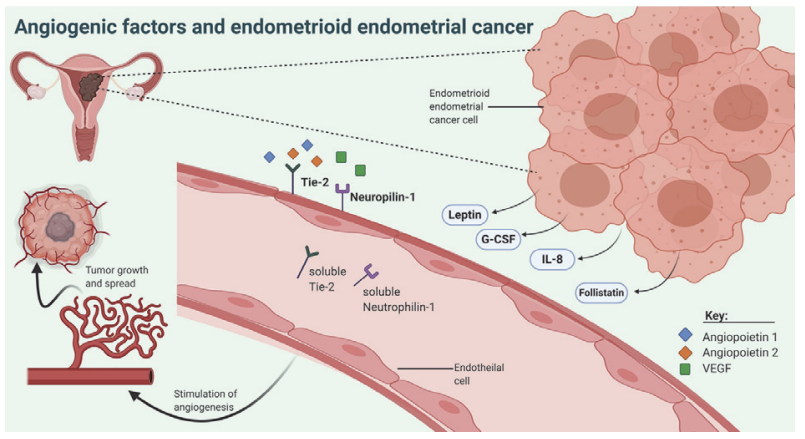

Abstract 622 Figure 1
B

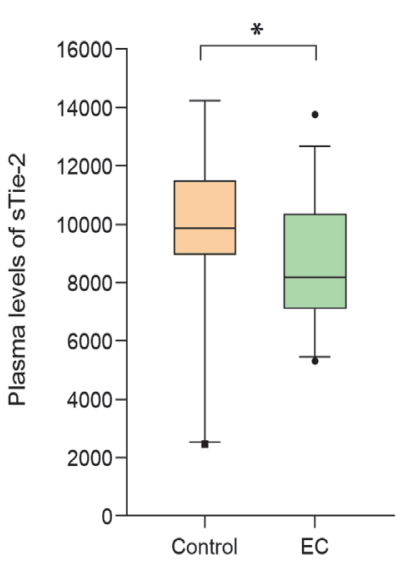

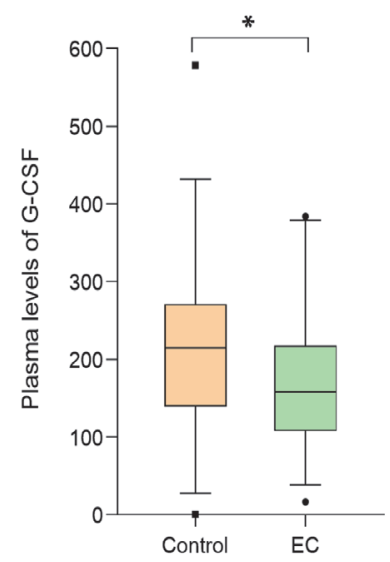

C

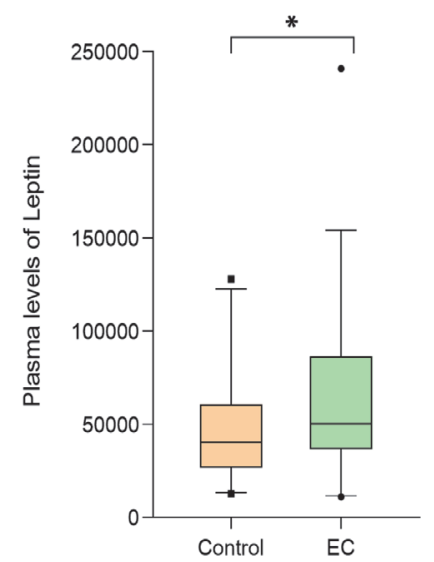

Abstract 622 Figure 2 
simultaneous determination of 37 different proteins with the Luminex $\mathrm{xMAP}^{\mathrm{TM}}$ multiplexing technology.

Result(s)* The case group comprised 38 patients with endometrioid EC, with mean age $65.9 \pm 8.2$ years and mean body mass index (BMI) of $31.8 \pm 6.1 \mathrm{~kg} / \mathrm{m}^{2}$. Lymphovascular invasion (LVI) was present in five patients while deep myometrial invasion (DMI) was present in 12 patients. The control group included 38 patients with mean age $66.8 \pm 8.3$ years and mean BMI of $27.6 \pm 3.9 \mathrm{~kg} / \mathrm{m}^{2}$. There was a significant difference in the BMI distribution between the case and control group $(p<0.01)$. The plasma levels of $\mathbf{s T i e - 2}$ and G-CSF were significantly decreased in EC patients compared to those of control patients, while the plasma levels of leptin were significantly higher in EC patients. Within the EC group, Tie-2 levels were lower in patients with LVI and DMI; however, these differences did not reach statistical significance. Additionally, follistatin, IL-8 and neuropilin-1 were also showing promising results.

Conclusion* The results of our study indicate that the plasma levels of different AFs might be involved in the growth of endometrioid EC. The plasma levels of G-CSF, Tie-2, and leptin significantly differ between EC and control patients. The plasma concentrations of these AFs could represent an important additional diagnostic tool for the early detection and characterization of EC and could guide the decision-making regarding the extent of surgery. Further validation studies with larger patient numbers are currently ongoing.

\section{PERIOPERATIVE COMPLICATIONS AFTER ENDOMETRIAL CANCER SURGERY: IS IT A REASON FOR UNDERTREATING OLDER PATIENTS?}

A Luzarraga*, N Teixeira, R Luna Guibourg, P Español, C Soler, R Rovira Negre. hospital de la santa creu i de sant pau, gynecology and obstetrics, Barcelona, Spain

\subsection{6/ijgc-2021-ESG0.177}

Introduction/Background* Older patients (OP) usually present with more aggressive endometrial cancer (EC) at diagnosis. However, they often receive suboptimal surgical treatment compared with non-older patients (NOP). Undertreatment, along with the presence of more aggressive tumours can explain the worse prognosis of EC in OP.

There is limited evidence comparing perioperative outcomes between OP and NOP. The benefit of applying complex procedures to OP is still controverse. The goal of this study was to compare complications after surgical treatment of EC between NOP and OP.

Methodology This is a retrospective single-center observational study including women undergoing surgery for EC between 2010 and 2019. Patients were classified according to age as NOP (younger than 75 years) or OP (75 years or older). Basal characterisitcs of groups were compared using Chisquare, Fisher's exact tests, student T-tests or Mann-Whithney tests. Kaplan Meier analysis was used to evaluate survival.

\section{Abstract 632 Table 1}

\begin{tabular}{|c|c|c|c|c|}
\hline & $<75$ & $\geq 75$ & Total & p-value \\
\hline Surgical approach & & & & 0.04 \\
\hline Laparoscopy $(\mathrm{N}, \%)$ & $145(78,4 \%)$ & $85(88,5 \%)$ & $230(81,9 \%)$ & \\
\hline Laparotomy $(\mathrm{N}, \%)$ & $31(16,8 \%)$ & $6(6.3 \%)$ & $37(13.2 \%)$ & \\
\hline Vaginal $(N, \%)$ & $1(0.5 \%)$ & $2(2,1 \%)$ & $3(1,1 \%)$ & \\
\hline Conversion tolaparotomy (N, \%) & $8(4,3 \%)$ & $3(3,1 \%)$ & $11(3,9 \%)$ & \\
\hline Type of surgery $(N, \%)$ & & & & $<0,01$ \\
\hline HT + DA only & $75(40,5 \%)$ & $60(61,2 \%)$ & $135(47,5 \%)$ & \\
\hline+ LFD $-p$ & $22(11,9 \%)$ & $13(13,3 \%)$ & $35(12,4 \%)$ & \\
\hline + LFD-p + LFD-PA & $43(23,1 \%)$ & $9(9,2 \%)$ & $52(18,4 \%)$ & \\
\hline + LFD-p + LFD-PA+ Omentectomy & $42(22,7 \%)$ & $13(13,3 \%)$ & $55(19,4 \%)$ & \\
\hline + Omentectomy only & $3(1,6 \%)$ & $3(3,1 \%)$ & $6(2,1 \%)$ & \\
\hline \multicolumn{5}{|l|}{ Nodal dissection } \\
\hline Pelviclymphadenectomy (N, \%) & $107(57,8 \%)$ & $35(36,5 \%)$ & $142(50,5 \%)$ & $<0,01$ \\
\hline Pelvicnodes dissected (mean, SD) & $13,4(7,7)$ & $11,8(7,6)$ & $12,9(7,7)$ & 0,24 \\
\hline Aorticlymphadenectomy $(\mathrm{N}, \%)$ & $85(45,9 \%)$ & $22(22.9 \%)$ & $107(38,1 \%)$ & $<0,01$ \\
\hline Aorticnodes dissected (mean, SD) & $10,6(7,9)$ & $6,7(6,3)$ & $9,5(7,7)$ & 0,01 \\
\hline Duration of surgery (mean, SD) & $175,4(89,2)$ & $152,2(76,1)$ & $167,4(85,4)$ & 0,03 \\
\hline Estimated blood loss (median, min-max) & $150(10-2500)$ & $100(10-2500)$ & $100(10-2500)$ & 0,30 \\
\hline \multicolumn{5}{|l|}{ Complications $(N, \%)$} \\
\hline Intraoperative & $12(6,5 \%)$ & $12(12,4 \%)$ & $24(8,5 \%)$ & 0,12 \\
\hline Post-operative & $25(13,5 \%)$ & $20(20,6 \%)$ & $45(16,0 \%)$ & 0,13 \\
\hline Early post-operative & $22(11,9 \%)$ & $20(20,6 \%)$ & $42(14,9 \%)$ & 0,06 \\
\hline Late post-operative & $7(3,8 \%)$ & $1(1,1 \%)$ & $8(2,9 \%)$ & 0,27 \\
\hline Clavien-Dindo classification ( $N, \%)$ & & & & 0,51 \\
\hline 1 & $2(1,1 \%)$ & $1(1,1 \%)$ & $3(1,1 \%)$ & \\
\hline II & $14(7,7 \%)$ & $9(9,5 \%)$ & $23(8,3 \%)$ & \\
\hline III & $8(4,4 \%)$ & $7(7,4 \%)$ & $15(5,5 \%)$ & \\
\hline IV & $1(0,5 \%)$ & 0 & $1(0,4 \%)$ & \\
\hline V & 0 & $1(1,1 \%)$ & $1(0,4 \%)$ & \\
\hline Blood transfusion $(\mathrm{N}, \%)$ & $9(4,9 \%)$ & $8(8,2 \%)$ & $17(6,0 \%)$ & 0,30 \\
\hline Number of $\mathrm{CH}$ (median, min-max) & $2(1-6)$ & $2(1-4)$ & $2(1-6)$ & 0,67 \\
\hline Abdominal drainage $(\mathrm{N}, \%)$ & $121(68,4 \%)$ & $53(55,2 \%)$ & $174(63,7 \%)$ & 0,03 \\
\hline Time drainage removal (median, min-max) & $3(1-14)$ & $3(1-28)$ & $3(1-28)$ & 0,14 \\
\hline Need for reintervention $(N, \%)$ & $8(4,5 \%)$ & $4(4,3 \%)$ & $12(4,4 \%)$ & 1,00 \\
\hline Length of hospital stay (median, min-max) & $4(1-137)$ & $4(2-32)$ & $4(1-137)$ & 0,87 \\
\hline \multicolumn{5}{|l|}{ Disease-specific survival } \\
\hline 5-year DSS & $86,0 \%(0,4)$ & $66,9 \%(0,7)$ & & 0.02 \\
\hline
\end{tabular}

